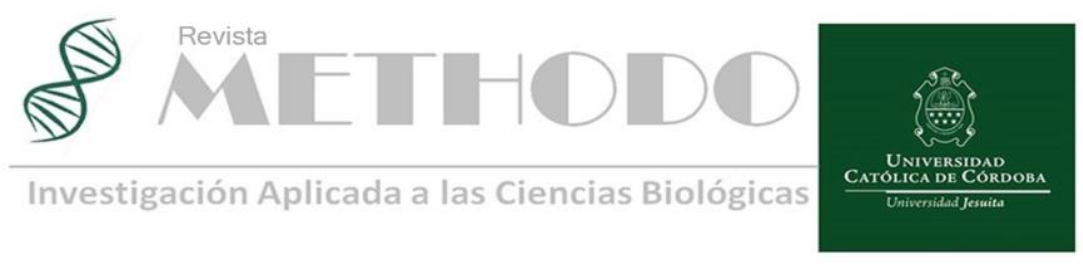

CASO CLINICO Rev. Methodo 2021;6(4):185-189 https://doi.org/10.22529/me.2021.6(4)06

Recibido 27 May. 2021| Aceptado 19 Jul. 2021|Publicado 05 Oct. 2021

\title{
Tratamiento no quirúrgico de la osteonecrosis relacionada a medicación asociada a un implante dental
}

\section{Conservative treatment of medication-related osteonecrosis of the jaw associated with a dental implant}

\author{
Verónica Flück $^{1}$ (D) , Claudia Analía Giacco ${ }^{1}$, Mariana Silvia Gandolfo $^{1}$ (D) , Lidia Isabel Adler ${ }^{1}$ (iD \\ 1. Universidad de Buenos Aires. Argentina, Facultad de Odontología, Cátedra de Clínica Estomatológica. \\ Correspondencia: Mariana S. Gandolfo, e-mail: marianasilviagandolfo@gmail.com
}

\section{Resumen}

La osteonecrosis de los maxilares es un serio efecto adverso asociado a medicamentos antirresortivos especialmente en pacientes con osteoporosis y neoplasias malignas. En este manuscrito, presentamos un caso clínico de una mujer caucásica de 56 años, con antecedentes de osteoporosis, medicada con bisfosfonatos, que cursó con osteonecrosis del maxilar inferior en relación con un implante dental. El tratamiento se basó fundamentalmente en la administración de terapia antibiótica, enjuagues bucales antibacterianos y en el tratamiento periodontal. El objetivo de ésta presentación fue comunicar un caso de osteonecrosis de la mandíbula alrededor de un implante dental relacionado con el uso de ácido ibandrónico tratado con éxito mediante un tratamiento conservador que incluía la aplicación local de minociclina.

Palabras clave: Osteonecrosis maxilar asociada a bifosfonatos, implante dental, clorhidrato de minociclina y tratamiento conservador.

\begin{abstract}
Osteonecrosis of the jaws is a serious adverse effect related with antiresorptive drugs, especially in patients with osteoporosis and malignant tumors. This article presents a case report of a 56-year-old caucasian woman with a history of osteoporosis treated with bisphosphonates who developed osteonecrosis of the lower jaw associated with a dental implant. Treatment was mainly based on the administration of antibiotic therapy, antibacterial mouthwashes and periodontal treatment. The aim of this paper was to report a case of osteonecrosis of the jaw around a dental implant related to the use of ibandronic acid successfully treated with conservative treatment including local application of minocycline.
\end{abstract}

Keywords: Bisphosphonate-associated osteonecrosis of the jaw, dental implant, minocycline hydrochloride and conservative treatment.

\section{Introducción}

La osteonecrosis maxilar asociada a medicación (ONMAM) se define en relación a las siguientes condiciones: uso actual o pasado de agentes

antirresortivos o antiangiogénicos, exposición del hueso maxilar o fístula intraoral o extraoral que persiste durante más de 8 semanas sin antecedentes de radioterapia de cabeza y cuello. La mayoría de los casos están precedidos por un procedimiento

Revista Methodo: Investigación Aplicada a las Ciencias Biológicas. Universidad Católica de Córdoba. Jacinto Ríos 571 Bo Gral. Paz. X5004FXS. Córdoba. Argentina. Tel.: (54) 3514517299 / Correo: methodo@ucc.edu.ar / Web: methodo.ucc.edu.ar | CASO CLINICO Rev. Methodo 2021;6(4):185-189. 
quirúrgico odontológico. En este contexto, la colocación de implantes dentales puede favorecer la aparición de la ONMAM ${ }^{1-5}$.

El aspecto anatomopatológico de la ONMAM es característico y se presenta con patrón histológico pagetoide, con signos de remodelación, disposición trabecular en mosaico, áreas de necrosis y colonias bacterianas en superficie y entre los espacios medulares ${ }^{6}$.

La presentación clínica es variable y puede mostrar distintos estadios. La American Association of Oral and Maxillofacial Surgeons (AAOMS) describió cuatro estadios clínicos, de acuerdo con la severidad de la patología. El estadio 0 incluye a los pacientes con síntomas inespecíficos o anomalías clínicas y radiográficas que pudieran deberse a la exposición a un agente antirresortivo sin evidencia clínica de hueso necrótico (variante no expuesta). El estadio 1 se caracteriza por la exposición de hueso necrótico en pacientes asintomáticos sin signos evidentes de infección. En el estadio 2 se observa la presencia de hueso necrótico expuesto en pacientes con síntomas y signos evidentes de infección y finalmente en el estadio 3 hay presencia de hueso necrótico expuesto en pacientes con dolor, infección y uno o más de los siguientes elementos: exposición de hueso necrótico con una extensión que supera la región del hueso alveolar, fractura patológica, fístula extraoral y/o comunicación bucosinusal o buconasal $^{7}$.

Las recomendaciones para el tratamiento de la ONMAM contemplan tanto el abordaje conservador, que incluye el tratamiento con antibióticos y/o analgésicos, el enjuague bucal con clorhexidina, y la eliminación de secuestros óseos, así como el abordaje invasivo o quirúrgico con el fin de eliminar el área de hueso necrótico ${ }^{7-11}$. Independientemente del tipo de abordaje terapéutico los antibióticos sistémicos, a pesar de su escasa penetración en el hueso necrótico, son utilizados frecuentemente y por períodos de tiempo prolongados en las lesiones de la ONMAM aumentando el riesgo de producir toxicidad sistémica $^{12}$. En este contexto, la aplicación local de antibióticos constituye una alternativa válida que permite sostener por un tiempo prolongado la concentración de la medicación en el sitio de la lesión evitando la toxicidad sistémica ${ }^{13}$.

La minociclina es un antibiótico de amplio espectro perteneciente al grupo de las tetraciclinas, que posee propiedades antiinflamatorias y un buen perfil de penetración en el tejido óseo ${ }^{14-16}$. La sustantividad de la minociclina en el hueso permite que este tejido actúe como un reservorio que mantiene la liberación de concentraciones efectivas del fármaco ejerciendo su acción antimicrobiana en las lesiones óseas durante un periodo de tiempo prolongado ${ }^{17}$. Además, la minociclina estimula a los osteoblastos a aumentar la matriz ósea ${ }^{18}$. En este sentido, el propósito del caso clínico presentado fue informar un enfoque alternativo exitoso al tratamiento no quirúrgico convencional con la aplicación local de microesferas de clorhidrato de minociclina en las lesiones de osteonecrosis de la mandíbula asociada a medicación en relación con los implantes dentales.

\section{Caso clínico}

Una mujer caucásica de 56 años se presentó a la consulta en la Cátedra de Clínica Estomatológica de la Facultad de Odontología manifestando dolor y sangrado en el lado izquierdo de la mandíbula con un tiempo de evolución de 2 semanas. La paciente tenía antecedentes de osteoporosis y tomaba ácido ibandrónico $150 \mathrm{mg}$ una vez al mes por vía oral desde hacía dos años. No presentaba otros antecedentes médicos de relevancia.

En la exploración clínica se observó la presencia de dos implantes colocados en el maxilar inferior, lado izquierdo, en los sectores correspondientes al segundo premolar y al primer molar en la zona precisamente donde la paciente refería dolor y sangrado. En la anamnesis la paciente refirió que dichos implantes habían sido colocados tres meses atrás. La rehabilitación protética no había sido realizada debido a los síntomas que presentaba.

La encía periimplantaria del implante situado en la región del segundo premolar estaba inflamada, con $9 \mathrm{~mm}$ de profundidad de bolsa. A la palpación, presentó dolor y drenó una secreción purulenta; sin embargo, no se observó movilidad de los implantes (Figura 1A y 1B).

Basándose en la historia clínica de la paciente y en las características clínico-radiológicas de la lesión bucal, se estableció el diagnóstico presuntivo de osteonecrosis de la mandíbula asociada a medicación. Se indicaron los siguientes estudios radiográficos: radiografía panorámica y tomografía computarizada de haz cónico (TC).

En la radiografía panorámica se observó reabsorción ósea en el hueso alveolar del cuello del implante. La TC reveló una estructura ósea trabecular irregular alrededor del implante afectado (Figura 1C y 1D).

De acuerdo con las características de la lesión descripta, el diagnóstico clínico establecido fue estadio 0 de osteonecrosis de la mandíbula asociada a medicación.

A partir de estas observaciones, y a pesar de que el tratamiento incluyó la terapia antibiótica (amoxicilina $500 \mathrm{mg}$ cada 8 horas hasta la remisión de la bolsa periodontal), el protocolo se centró en el cuidado local mediante enjuagues bucales que realizaba la paciente con una solución 
antimicrobiana (clorhexidina al $0,12 \%, 2$ veces al día) y, en las consultas de control, mediante el lavaje de la fístula y de la bolsa periodontal (digluconato de clorhexidina al $0,12 \%$ ) y el tratamiento periodontal.

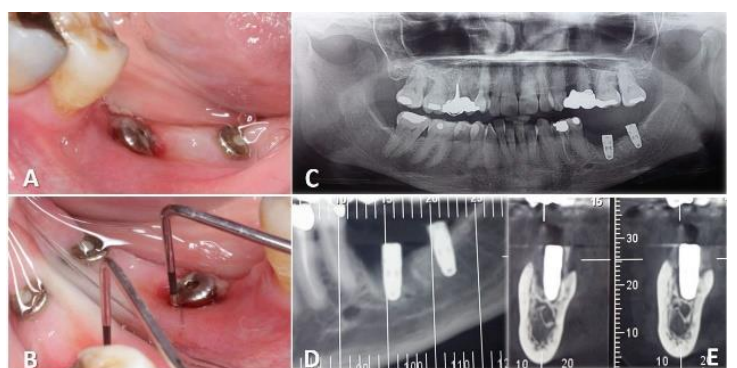

Figura 1. A) Imagen clínica inicial B) Bolsa de $9 \mathrm{~mm}$ asociada a sangrado al sondeo C) Radiografía que muestra reabsorción ósea alrededor del implante. D) y E): Imagen de TC alrededor del implante.

Durante el tratamiento periodontal, se eliminó un secuestro óseo de la bolsa periodontal (Figura 2A). Tras este procedimiento, se realizó una irrigación inicialmente con solución salina normal y después con clorhexidina al $0,12 \%$. El defecto óseo se secó con una gasa estéril y se colocaron localmente microesferas de clorhidrato de minociclina de 100 mg (Acneclin 100 AP) en la bolsa periodontal para conseguir un efecto antibacteriano $y$ antiinflamatorio. El material extraído de la bolsa periodontal era un fragmento irregular de tejido óseo duro y marrón. Se realizó el análisis histopatológico del mismo. Los cortes histológicos revelaron la presencia de trabéculas necróticas con signos de remodelación y un aspecto similar al de la enfermedad de Paget. Los espacios de la médula habían sido reemplazados por detritus celulares y colonias microbianas que incluían elementos fúngicos filamentosos (Figura 2B). El diagnóstico histopatológico establecido fue osteonecrosis asociada a infección fúngica.

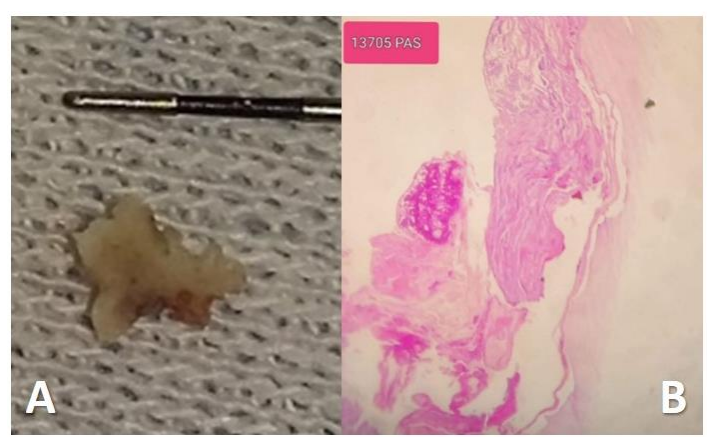

Figura 2. A) Secuestro óseo. B) Imagen histológica (H\&E, x 40).

En el control de seguimiento, tras un mes de toma de antibióticos y tratamiento local, la medida de la profundidad de la bolsa se redujo significativamente y no se observó sangrado ni supuración durante el procedimiento (Figura 3). Actualmente, tras un año de seguimiento mensual, la profundidad de la bolsa se mantiene en 3 milímetros.

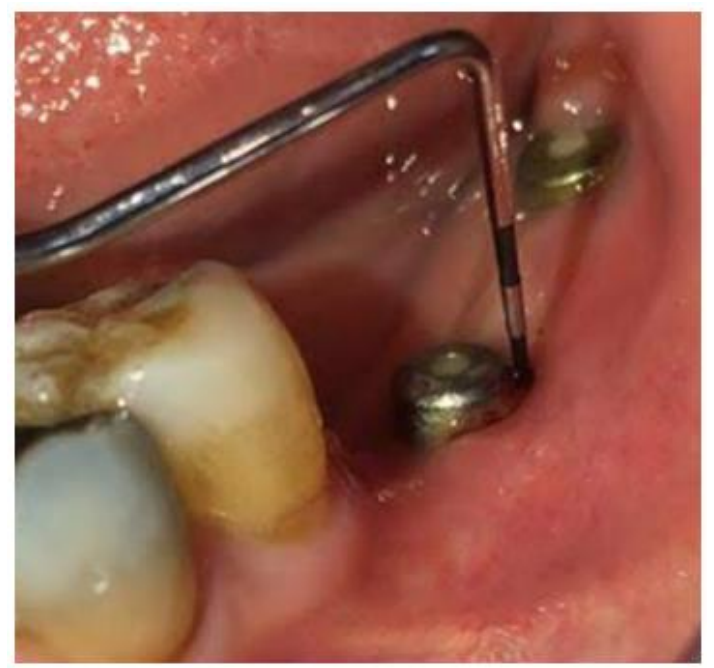

Figura 3. Visita de seguimiento de 1 mes. Valor de la profundidad de sondeo: $3 \mathrm{~mm}$.

\section{Discusión}

La ONMAM se define como la presencia de hueso necrótico expuesto en la región maxilofacial, que se mantiene durante un período mínimo de 8 semanas, sin antecedente de radioterapia en la zona. Esta condición es infrecuente y ha sido asociada a dos grupos de medicamentos: los antirresortivos y los antiangiogénicos $7,19,20$.

Kwon et al. (2014) informaron que la aparición de la ONMAM desencadenada por la cirugía de implantes dentales suele producirse en un lapso de tiempo inferior a 6 meses después del procedimiento y puede estar asociada a la invasión quirúrgica ${ }^{1}$. Además, en 2014, Tam et al. presentaron dos casos de osteonecrosis asociada a implantes en pacientes sometidos a tratamiento con bifosfonatos orales y propusieron que el traumatismo quirúrgico durante la cirugía de implantes podría estimular la acumulación postoperatoria del fármaco en el lugar del implante ${ }^{21}$.

Nuestra paciente presentó una ONMAM tres meses después de la colocación de los implantes. Según la clasificación de Kwon et al (2014), el patrón de osteonecrosis en este caso fue "en bloque"1. El traumatismo quirúrgico en el hueso alveolar durante la cirugía de los implantes podría haber estimulado aún más la acumulación postoperatoria del fármaco en el lugar del implante, causando osteonecrosis, ya que el ácido ibandrónico no se interrumpió en nuestra paciente. La literatura muestra que la resolución completa de la ONMAM se produce con mayor frecuencia a través de la cirugía ${ }^{11,22}$. Además, los estudios sobre

Revista Methodo: Investigación Aplicada a las Ciencias Biológicas. Universidad Católica de Córdoba. Jacinto Ríos 571 Bo Gral. Paz. X5004FXS. Córdoba. Argentina. Tel.: (54) 3514517299 / Correo: methodo@ucc.edu.ar / Web: methodo.ucc.edu.ar | CASO CLINICO Rev. Methodo 2021;6(4):185-189. 
el tratamiento no quirúrgico de la enfermedad han sido menos eficaces y muy escasos ${ }^{23}$.

La estrategia de tratamiento para los pacientes en estadios tempranos (estadios 0 y 1 ) incluye: tratamiento local conservador, tratamiento sistémico con analgésicos y antibióticos y el enjuague bucal antibacteriano ${ }^{20}$.

En relación con el enfoque terapéutico conservador, en la literatura existe un único informe sobre el tratamiento no quirúrgico de la ONMAM que incluye la minociclina tópica dentro del protocolo terapéutico. En este trabajo se informaron resultados favorables utilizando un protocolo modificado con minociclina tópica en Orabase para tratar las lesiones de $\mathrm{ONMAM}^{24}$. En este reporte de caso hemos propuesto un enfoque alternativo y satisfactorio al tratamiento no quirúrgico convencional mediante la aplicación local de microesferas de clorhidrato de minociclina en las lesiones de ONMAM relacionadas con implantes dentales. Aunque el protocolo terapéutico aplicado fue eficaz, no podemos ignorar que la remisión completa de la ONMAM y la conservación del implante fueron posibles porque la osteonecrosis afectó una zona limitada alrededor del implante. Por lo tanto, la aplicación de este protocolo combinado podría tener éxito para el tratamiento de los estadios tempranos (estadios 0 y 1) de la ONMAM asociada a los implantes dentales. Por otra parte, es importante resaltar la relevancia del manejo interdisciplinario médico-odontológico que debe incluir una exhaustiva anamnesis y una detallada historia clínica con el fin de evitar complicaciones en la atención y seguimiento de los pacientes medicados con agentes antirresortivos o antiangiogénicos.

\section{Bibliografía}

1. Kwon TG, Lee CO, Park JW, Choi SY, et al. Osteonecrosis associated with dental implants in patients undergoing bisphosphonate treatment. Clin Oral Implants Res. 2014 May;25(5):632-40. doi: 10.1111/clr.12088.

2. Starck WJ, Epker BN. Failure of osseointegrated dental implants after diphosphonate therapy for osteoporosis: a case report. Int $\mathbf{J}$ Oral Maxillofac Implants. 1995 Jan-Feb;10(1):74-8. PMID: 7615320.

3. Jacobsen C, Metzler P, Rössle M, Obwegeser $\mathrm{J}$, et al. Osteopathology induced by bisphosphonates and dental implants: clinical observations. Clin Oral Investig. 2013 Jan;17(1):167-75. doi: 10.1007/s00784-0120708-2.

4. Kuroshima S, Sasaki M, Sawase T. Medication-related osteonecrosis of the jaw:
A literature review. J Oral Biosci. 2019 Jun;61(2):99-104. doi: 10.1016/j.job.2019.03.005.

5. Marx RE. Pamidronate (Aredia) and zoledronate (Zometa) induced avascular necrosis of the jaws: a growing epidemic. J Oral Maxillofac Surg. 2003 Sep;61(9):11157. doi: 10.1016/s0278-2391(03)00720-1.

6. Paparella ML, Brandizzi D, Santini-Araujo E, Cabrini RL. Histopathological features of osteonecrosis of the jaw associated with bisphosphonates. Histopathology. 2012 Feb;60(3):514-6. doi: 10.1111/j.13652559.2011.04061. $\mathrm{x}$.

7. Ruggiero SL, Dodson TB, Fantasia J, Goodday R, et al. American Association of Oral and Maxillofacial Surgeons position paper on medication-related osteonecrosis of the jaw--2014 update. J Oral Maxillofac Surg. 2014 Oct;72(10):1938-56. doi: 10.1016/j.joms.2014.04.031. Epub 2014 May 5. Erratum in: J Oral Maxillofac Surg. 2015 Jul;73(7):1440. Erratum in: J Oral Maxillofac Surg. 2015 Sep;73(9):1879.

8. Marx RE, Sawatari Y, Fortin M, Broumand V. Bisphosphonate-induced exposed bone (osteonecrosis/osteopetrosis) of the jaws: risk factors, recognition, prevention, and treatment. J Oral Maxillofac Surg. 2005 Nov;63(11):1567-75. doi: 10.1016/j.joms.2005.07.010.

9. Voss PJ, Joshi Oshero J, Kovalova-Müller A, Veigel Merino EA, et al. Surgical treatment of bisphosphonate-associated osteonecrosis of the jaw: technical report and follow up of 21 patients. J Craniomaxillofac Surg. 2012 Dec;40(8):719-25. doi: 10.1016/j.jcms.2012.01.005. Epub 2012 Feb 14. PMID: 22336489.

10. Rupel K, Ottaviani G, Gobbo M, Contardo L, et al. A systematic review of therapeutical approaches in bisphosphonates-related osteonecrosis of the jaw (BRONJ). Oral Oncol. 2014 Nov;50(11):1049-57. doi: 10.1016/j.oraloncology.2014.08.016.

11. Hayashida S, Soutome S, Yanamoto S, Fujita S, et al. Evaluation of the Treatment Strategies for Medication-Related Osteonecrosis of the Jaws (MRONJ) and the Factors Affecting Treatment Outcome: A Multicenter Retrospective Study with Propensity Score Matching Analysis. J Bone Miner Res. 2017 Oct;32(10):2022-2029. doi: 10.1002/jbmr.3191. 
12. Ruszczak Z, Friess W. Collagen as a carrier for on-site delivery of antibacterial drugs. Adv Drug Deliv Rev. 2003 Nov 28;55(12):167998. doi: 10.1016/j.addr.2003.08.007.

13. Zalavras CG, Patzakis MJ, Holtom P. Local antibiotic therapy in the treatment of open fractures and osteomyelitis. Clin Orthop Relat Res. $2004 \quad$ Oct;(427):86-93. doi: 10.1097/01.blo.0000143571.18892.8d.

14. Javed S, Kohli K. Local delivery of minocycline hydrochloride: a therapeutic paradigm in periodontal diseases. Curr Drug Deliv. 2010 Dec;7(5):398-406. doi: $10.2174 / 156720110793566290$.

15. Xu Y, Wei W. A comparative study of systemic subantimicrobial and topical treatment of minocycline in experimental periodontitis of rats. Arch Oral Biol. 2006 Sep;51(9):794-803. doi: 10.1016/j.archoralbio.2006.03.018.

16. Garrido-Mesa N, Zarzuelo A, Gálvez J. Minocycline: far beyond an antibiotic. $\mathrm{Br} \mathrm{J}$ Pharmacol. 2013 May;169(2):337-52. doi: 10.1111/bph.12139.

17. Torabinejad M, Khademi AA, Babagoli J, Cho Y, et al. A new solution for the removal of the smear layer. J Endod. 2003 Mar;29(3):170-5. doi: 10.1097/00004770200303000-00002.

18. Gomes PS, Fernandes MH. Effect of therapeutic levels of doxycycline and minocycline in the proliferation and differentiation of human bone marrow osteoblastic cells. Arch Oral Biol. 2007 Mar;52(3):251-9.

doi: 10.1016/j.archoralbio.2006.10.005.
19. Khan AA, Morrison A, Hanley DA, Felsenberg D, et al. Diagnosis and management of osteonecrosis of the jaw: a systematic review and international consensus. J Bone Miner Res. 2015 Jan;30(1):3-23. doi: 10.1002/jbmr.2405.

20. Kim KM, Rhee Y, Kwon YD, Kwon TG, et al. Medication Related Osteonecrosis of the Jaw: 2015 Position Statement of the Korean Society for Bone and Mineral Research and the Korean Association of Oral and Maxillofacial Surgeons. J Bone Metab. 2015 Nov;22(4):151-65. 10.11005/jbm.2015.22.4.151

21. Tam Y, Kar K, Nowzari H, Cha HS, et al. Osteonecrosis of the jaw after implant surgery in patients treated with bisphosphonates--a presentation of six consecutive cases. Clin Implant Dent Relat Res. 2014 Oct;16(5):75161. doi: 10.1111/cid.12048.

22. Rupel K, Ottaviani G, Gobbo M, Contardo L, et al. A systematic review of therapeutical approaches in bisphosphonates-related osteonecrosis of the jaw (BRONJ). Oral Oncol. 2014 Nov;50(11):1049-57. doi: 10.1016/j.oraloncology.2014.08.016.

23. Fliefel R, Tröltzsch M, Kühnisch J, Ehrenfeld $\mathrm{M}$, et al. Treatment strategies and outcomes of bisphosphonate-related osteonecrosis of the jaw (BRONJ) with characterization of patients: a systematic review. Int $\mathrm{J}$ Oral Maxillofac Surg. 2015 May;44(5):568-85. doi: 10.1016/j.ijom.2015.01.026.

24. Karasneh JA, Al-Eryani K, Clark GT, Sedghizadeh PP. Modified protocol including topical minocycline in orabase to manage medication-related osteonecrosis of the jaw cases. J Oral Pathol Med. 2016 Oct;45(9):718720. doi: 10.1111/jop.12419.

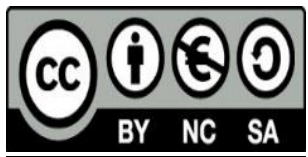

